\title{
Rajan Jain and Marco Essig (Eds): Brain Tumor Imaging
}

\author{
Thieme Medical Publishers, 2016. ISBN 978-1-60406-806-1
}

\author{
Emanuela Mazziotti ${ }^{1} \cdot$ Luigi Mansi $^{1}$
}

Published online: 14 May 2016

(C) Springer-Verlag Berlin Heidelberg 2016

This book is a practical and comprehensive manual on the most important imaging modalities used in neurooncology. Written with the contribution of many international experts, it is edited by two well-known neuroradiologists: Rajan Jain, Associate Professor of Radiology at New York University, and Marco Essig, Professor and Chairman of Radiology at the University of Manitoba.

The book is presented as an essential guide to the most advanced imaging techniques, both morphological and functional, used in clinical practice for the diagnosis and follow-up of patients with brain tumours. The text consists of 19 chapters, nearly all of which are based on magnetic resonance imaging (MRI) and its main applications. As early as in the first two chapters the authors state the superiority of MRI both for the study of brain tumours and for the evaluation of response to treatments (RANO criteria).

Over the next ten chapters, the most relevant functional imaging techniques are highlighted. In particular, chapter $4-7$ discuss perfusion studies including both the three main techniques of MRI (DSC-MRP, DCE-MRP, MRP-ASL) and the limitations and advantages of perfusion computed tomography (CT), evaluating their role in the differential diagnosis of cerebral lesions, in the staging of gliomas, and in the differential diagnosis of recurrence. The chapter 8 and 9 are devoted to magnetic resonance diffusion and its techniques, isotropic (DWI) and anisotropic (DTI), while the chapter 10 describes the importance of fMRI and DTI tractography for the noninvasive assessment and mapping of brain areas. The chapter 11 focuses on magnetic resonance spectroscopy and its roles in treatment planning and in

Luigi Mansi

luigi.mansi@unina2.it

Second University of Naples, Naples, Italy monitoring response in neurooncological patients, both adults and children. The section on functional imaging ends with chapter 12. This chapter is devoted to nuclear medicine techniques (SPECT, PET, PET/CT and PET/MRI), which may provide a significant contribution to diagnosis, staging, and therapy planning, with an important role also in the differentiation between recurrent tumour and the effects of treatment in patients with brain tumours. Chapter 13 is devoted to the imaging modalities used to evaluate the effects of treatment and to differentiate them from a true neoplastic progression, and chapter 14 provides a deeper discussion of the diagnostic procedures aimed at the early identification of neurological complications of cancer (such as cerebrovascular accidents and cerebral paraneoplastic syndromes), which significantly alter morbidity and mortality. Finally, after a brief discussion on the historical and current applications of intraoperative magnetic resonance in chapter 15 , the last four chapters deal with methods still being studied that open new horizons in neurooncological imaging.

All the chapters are accompanied by many pictures, in colour and in black and white, all with extensively comments, and by tables and explanatory graphs of the content of the text. The many high-quality images, always helpful and effective, the clear language, the strict approach which combines a conversational tone with a great wealth of detailed information make this manual appropriate for radiologists, neuroradiologists, neurooncologists, neurosurgeons and all physicians involved in taking care of patients with brain tumours. Amongst this group of readers, nuclear physicians may find a particular interest in the book, which more than deepens their knowledge of procedures in providing a better understanding of their indications in the whole scenario in which at present MRI plays a major role. A wider analysis of the possible contribution of radiotracers beyond FDG or of the innovative approaches allowed by PET/MRI is awaited with the hope that they will find a role in daily clinical practice. 